\title{
Dietary intake of energy, nutrients and phenolic compounds in Italian primary school children and their environmental impact
}

\section{Abstract}

Data from observational and interventional studies demonstrate the key role of diet on both human health and environmental sustainability. However, there is a lack of studies investigating the environmental impact of children's dietary behavior. In this regard, detailed information on population-level intakes is required to translate these findings into possible recommendations.

The aim of this study was to investigate the nutritional composition of the diet and its related environmental impact in a sample of Italian primary school children $(\mathrm{n}=200,8-11$ years, $52 \% \mathrm{~F}-48 \% \mathrm{M})$.

Children completed a 3-day food record twice, in winter and spring. Dietary records were processed to obtain mean daily data on food group intakes. Mean daily energy and nutrient intakes were computed applying the Italian food database of the European Institute of Oncology, while (poly)phenolic compound contents were estimated from the Phenol-Explorer or by specific searches in the most up to date scientific literature. The mean daily environmental impact was estimated through the Barilla Center for Food and Nutrition environmental database taking into account the carbon footprint and ecological footprint. Children's BMI was calculated by measuring height and weight at the beginning of each assessment week.

No differences in children's BMI as well as in the consumption of food groups were found between the two seasons. The intra-individual variability was low but a high inter-individual variability was registered. Energy and nutrient intakes were similar between the two seasons and were found to be in line with national data referred to school age children. Adherence to the Italian nutritional recommendations (LARN) was not satisfied for total fats, sugars, and some vitamins and minerals. The main contributors to the phenolic intake were flavonoids and phenolic acids, while the most representative subclasses were flavan-3-ols and hydroxycinnamic acids. Main dietary sources of (poly)phenolic compounds were fruit juices, apples, pears, potatoes, and tomatoes. Carbon and ecological footprints were higher during winter and the main dietary contributors were red meat; milk, yogurt and dairies; pizza and focaccia, for both indexes.

This study provided the first comprehensive analysis of the nutritional composition and the environmental impact of Italian primary school children diet. Further research is needed to evaluate children habitual diet for investigating a possible relationship between health and environmental sustainability. Moreover, school education programs have to be implemented for promoting healthy and sustainable dietary behaviors.

\section{Conflict of Interest}

There is no conflict of interest. 